\title{
Fluorescence-Guided Resection of Glial Brain Tumors with Fotoditazin
}

\author{
Artemii Yurievich Rynda*, Dmitrii Michailovich Rostovtsev, Victor Emelijanovich Olyushin, \\ Yliay Michaiylovna Zabrodskaya
}

Department Neurooncology, Polenov Russian Research Institute of Neurosurgery, Div. Almazov National Medical Research Centre, St. Petersburg, Russia

\author{
Email address: \\ artemii.rynda@mail.ru (A. Y. Rynda), zabrjulia@yandex.ru (Y. M. Zabrodskaya), Fed_56@mail.ru (V. E. Olyushin), \\ dok79@mail.ru (D. M. Rostovtsev) \\ ${ }^{*}$ Corresponding author
}

\section{To cite this article:}

Artemii Yurievich Rynda, Dmitrii Michailovich Rostovtsev, Victor Emelijanovich Olyushin, Yliay Michaiylovna Zabrodskaya. Fluorescence-Guided Resection of Glial Brain Tumors with Fotoditazin. Journal of Surgery. Vol. 6, No. 5, 2018, pp. 116-122. doi: $10.11648 /$ j.js.20180605.12

Received: July 24, 2018; Accepted: August 15, 2018; Published: September 12, 2018

\begin{abstract}
Objective: the purpose of this study was to assess the usefulness and accuracy of visualization of glial brain tumors of varying degrees of malignancy when surgically removed with fluorescent control of fotoditazine. Evaluation of the edges of tumor tissue was also carried out in order to increase the degree gross total resection (GTR), and to evaluate the specificity and sensitivity of the fluorescence method. Design and methods: thirty one glial tumor patients, with varying degrees of malignancy, underwent controlled fluorescence-guided resection in the presence of the indicator molecule fotoditazine. To detect fluorescence, a OHS-1 operating microscope Leica with a special fluorescence module was used. Evaluation of the efficacy, sensitivity and specificity of the method was assessed using various histo-morphological studies. GTR was assessed using postoperative MRI. Results: for grade I and II gliomas, the sensitivity of the surgical tumor removal method was $68.3 \%$, and the specificity was $60.1 \%$. For grade III and IV gliomas, the sensitivity of the surgical tumor removal method was $85.4 \%$, and the specificity was $76.2 \%$. The extent to which total surgical resection was achieved was $79.3 \%$ in grade I-II and $95.6 \%$ in grade III-IV. Conclusions: intraoperative fluorescent imaging with fotoditazine is a highly effective, sensitive, and specific method which permits glial tumors of various histologic types to be resected more completely and effectively.
\end{abstract}

Keywords: Fluorescence-Guided Resection, Glial Tumor, Fluorescence, Photodiagnostics, Fotoditazin

\section{Introduction}

In addition to patient age, functional status, tumor localization, and the degree of malignancy, one of the main factors determining patient outcome is the completeness of tumor removal [1]. Total removal of gliomas is a practically impossible task due to infiltrative growth, which often involves spread into functionally significant zones [2]. Various methods are used in order to identify glioma boundaries during brain surgery. Examples include intraoperative neuronavigation, ultrasound navigation, and MRI. In terms of tumor resection, intraoperative photodiagnosis is not simply an additional method; it may, in fact, be the best method [3-8]. Many authors report that fluorescence-controlled resection permits more thorough tumor removal and improves quality of life compared to traditional glioma surgery [9-11].

The use of intraoperative photodiagnostics in glioma cases has several advantages: more intense visualization of anaplastic sites with gliomas; better tissue differentiation with continuing high grade gliomas after previous combined treatment [12]; the possibility of detecting tumor cells in ventricular walls, even in the absence of visible invasion of their walls; and improved degree of tumor resection [13]. 
Several authors have evaluated fluorescently controlled glioma resection, by comparing it with histomorphological results, and concluded that it compares favorably [14-17].

Purpose

The aim of this work is to improve the radical resection method and its outcomes in glial brain tumors. The authors also determined the sensitivity and specificity of fluorescence-guided resection using the photosensitizer fotoditazin.

\section{Materials and Methods}

\subsection{Study Design}

Surgeries were performed at the Russian Polenov Research Institute for Neurosurgery (Department of Neurooncology) from October 2011 to December 2015. Specifically, 31 patients with glial tumors of supratentorial localization and various degrees of malignancy underwent intraoperative fluorescence-controlled resection. In order to induce visible fluorescence, a second generation reagent in the E6 group of compounds, namely fotoditazine, was used. Tumor evaluation was performed according to the World Health Organization's Histological Classification of Tumours of the Central Nervous System (2016) [18]. Following the administration of an initial anesthesia, patients on the operating table were intravenously given fotoditazine (VetaGrand, Russia) 1.5-2.0 hours before the expected removal of the tumor tissue. Preparations were diluted in saline $(200 \mathrm{ml})$ such that the active ingredient, chlorin E6, was $1 \mathrm{mg}$ per $1 \mathrm{~kg}$ of patient body weight. Fotoditazine selectively accumulates in tumor tissue, yet concentrates minimally in normal brain tissue. Thus, the characteristic red fluorescence of chlorins identify tumor areas of the brain. In order to conduct the research at fluorescent wavelengths, an OHS-1 operating microscope (Leica, Germany) was coupled to a fluorescent accessory (LOMO Ltd, Russia). Fluorescent imaging allows the investigating physician to define, with high color contrast, the tumor tissues that have accumulated fotoditazine, relative to tissues into which the drug has not penetrated (Figure 1, 2, 3, 4, 5).

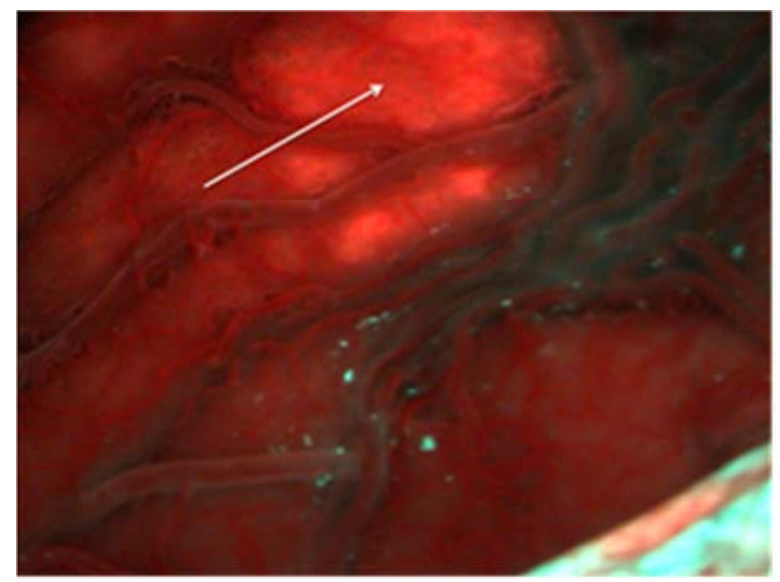

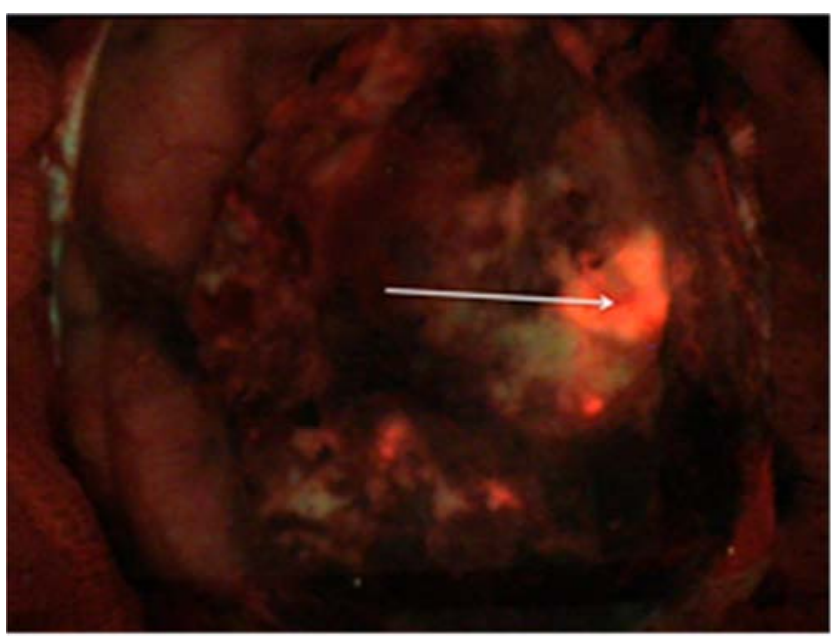

Figure 2. Fluorescence of fotoditazine in anaplastic oligoastrocytoma.

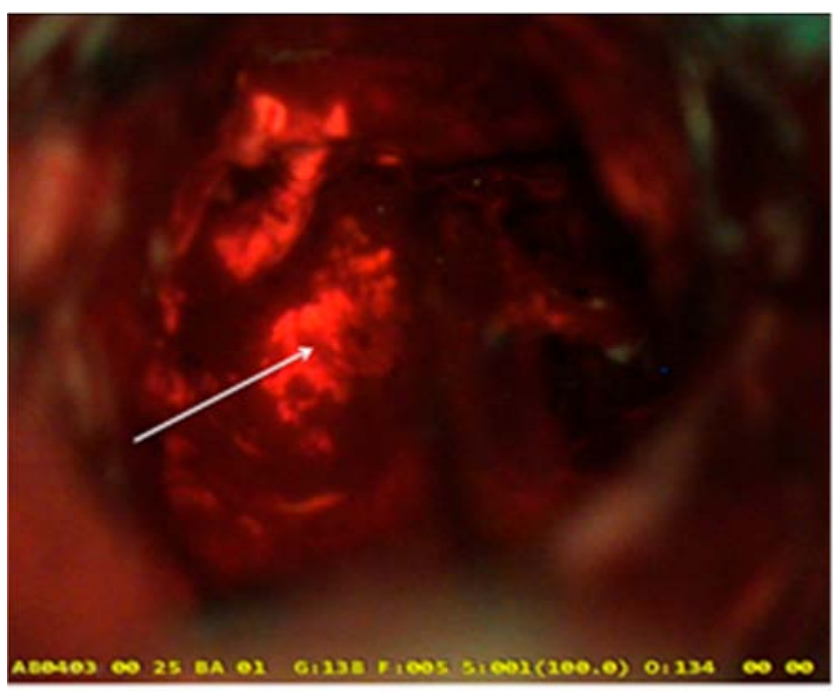

Figure 3. Fluorescence of fotoditazine in anaplastic astrocytoma

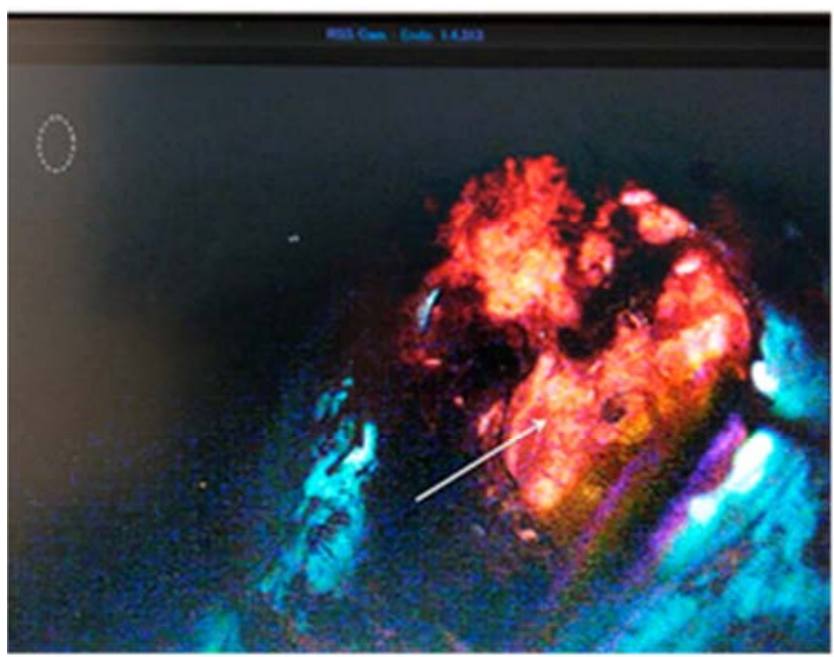

Figure 4. Fluorescence of fotoditazine in glioblastoma.

Figure 1. Fluorescence of fotoditazine in diffuse astrocytoma, seen through unchanged temporal lobe cortex. 


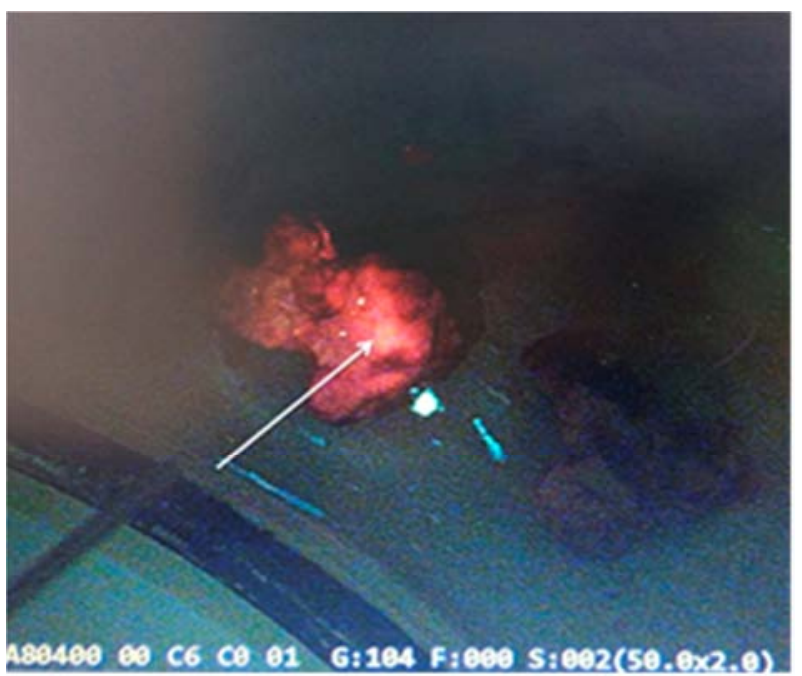

Figure 5. Fluorescence of fotoditazine in a gliosarcoma node.

During surgery, biopsies were taken from various regions of the tumor, depending on the degree of fotoditazine fluorescence, and processed by histological methods. In order to prevent post-operative photochemical retinal damage (burns), patients wore protective eyewear for 24 hours following surgery.

\subsection{Ethical Statement}

This survey was conducted in compliance with the protocol Good Clinical Practices and Declaration of Helsinki principles. Written informed consent to participate in the study after being informed about the purpose of study was given by all included patients. Investigators guaranteed the total anonymity of the information derived from the research.

\subsection{Statistical Analysis}

Statistical processing of data was carried out using the STATISTICA v.10 software package (StatSoft Inc., USA). For continuous variables, the median and quartiles (LQ, UQ) were calculated. Qualitative variables are represented in the form of the absolute number of observations. A pair-wise comparison of dependent samples was carried out using the Wilcoxon test. Comparison of independent groups by quantitative traits was carried out using the Student's t-test. According to the Bonferroni principle, $\mathrm{P}$ values of less than 0.01 were taken as statistically significant for multiple comparisons. The study was approved by the Ethics Committee of the Polenov Russian Research Institute of Neurosurgery and implemented in accordance with the principles of the Helsinki Declaration.

\section{Results}

The effectiveness of fluorescent diagnostics was evaluated according to the following:

1. comparison of visual fluorescence assessments with histological data from fluorescent and non-fluorescent tissues
2. comparison of contrast MRI with the results of intraoperative fluorescence

3. comparison of the distribution and accumulation of fotoditazine in tumor and normal brain tissue, depending on the morphological diagnosis

Patients were classified into 4 groups, depending on the degree of tumor malignancy. The World Health Organization's Histological Classification of Tumours of the Central Nervous System (2016) specifies four grades, and the distribution of research subjects, according to that scheme, are shown in Table. 1.

Table 1. Visible intraoperative fluorescence prior to resection, by Grade.

\begin{tabular}{lll}
\hline \multirow{2}{*}{ Grade } & Fluorescence & \\
\cline { 2 - 3 } & positive & negative \\
\hline I $(n=2)$ & $1(50.0 \%)$ & $1(50.0 \%)$ \\
II $(n=5)$ & $4(80.0 \%)$ & $1(20.0 \%)$ \\
III $(n=9)$ & $8(88.9 \%)$ & $1(11.1 \%)$ \\
IV $(n=15)$ & $14(93.3 \%)$ & $1(6.7 \%)$ \\
\hline
\end{tabular}

The patients in these WHO glioma categories can be further characterized. In Grade I, there were $2(100 \%)$ pilocytic astrocytomas. In Grade II, there were $2(40 \%)$ diffuse astrocytomas, $2(40 \%)$ oligoastrocytomas, and 1 (20\%) oligodendroglioma. In Grade III, there were 6 (66.7\%) anaplastic astrocytomas, $1 \quad(11.1 \%)$ anaplastic oligodendroglioma, and $2 \quad(22.2 \%)$ anaplastic oligoastrocytomas. In Grade IV, there were $13(86.7 \%)$ glioblastomas, and $2(13.3 \%)$ gliosarcomas. A 4-point scale was used for visual assessment of fluorescence intensity: $0=$ no visible fluorescence $(-) ; 1=\operatorname{dim}$ red light $(+) ; 2=$ red glow $(++) ; 3=$ bright red glow $(+++)$. The results are shown in Table 2 .

Table 2. Intensity of visible intraoperative fluorescence prior to resection, by Grade.

\begin{tabular}{llllll}
\hline Grade & \multicolumn{6}{l}{ Intensity of fluorescence } & Total \\
\cline { 2 - 6 } Tumors & $\mathbf{0}$ & $\mathbf{1}$ & $\mathbf{2}$ & $\mathbf{3}$ & 2 \\
\hline Grade I & 1 & - & 1 & - & 5 \\
Grade II & 1 & 1 & 2 & 1 & 9 \\
Grade III & 1 & 1 & 1 & 6 & 15 \\
Grade IV & 1 & - & 1 & 13 & $31(100 \%)$ \\
Total & $4(12.9 \%)$ & $2(6.5 \%)$ & $5(16.1 \%)$ & $20(64.5 \%)$ & 3 \\
\hline
\end{tabular}

A significant correlation was seen between the degree of tumor malignancy and the intensity of visible fluorescence ( $p$ $<0.05$ ). Visible fluorescence was much more frequent in the Grade III-IV tumor group, compared to gliomas in the Grade I-II group. Among Grade I-II gliomas, visible fluorescence was seen in 5 out of the 7 patients (71.4\%). Among Grade III-IV gliomas cases, visible fluorescence was seen in 22 out of the 24 patients $(91.7 \%)$. Estimation of residual fluorescence was carried out throughout the entire glial tumor removal process, and intraoperative video recordings were used for data analysis.

In the group who underwent surgery (100\%), 69\% displayed no visible fluorescence after the intervention, yet $31 \%$ still displayed residual fluorescence. In the latter group ( $31 \%$ group), a decision was made to further increase the 
resection in an attempt to improve outcomes; in some cases, additional resection in was not considered advisable due to the spread of the tumor to functionally significant areas, therefore 5\% underwent further resection. Neurologic deficits were not observed in connection with the additional resection procedure described above $\left(5 \%\right.$ group, $2^{\text {nd }}$ resection following intraoperative illumination). It should be pointed out that all patient received fotoditazine and even patients not receiving additional resection were treated with photodynamic therapy (PDT) in the hope that tumor photosensitized cells would be induced into apoptosis [19].

Comparative analysis of visible fluorescence intensity in all of the glioma groups, using variance analysis and Student's t-test methods, revealed statistically significant

differences between Grade II-Grade III gliomas, Grade IIGrade IV gliomas and between Grade III-Grade IV gliomas $(p=0.003)$. In a comparative analysis of visible fluorescence with preoperative contrast MRI data, a direct correlation was seen with the degree of glioma malignancy $(p>0.01)$. The pattern was: the higher the malignancy, the greater the degree of contrast according to the MRI data, and the brighter the visible fluorescence during the surgery. Comparative analysis of visible fluorescence with histological examination data (Ki-67, p53) revealed a significant correlation with glioma malignancy (Spearman coefficient $\mathrm{p}<0.0001$ ). A brighter fluorescence was observed with Grade IV gliomas; weak fluorescence was more marked in low-grade gliomas (Figure $6)$.

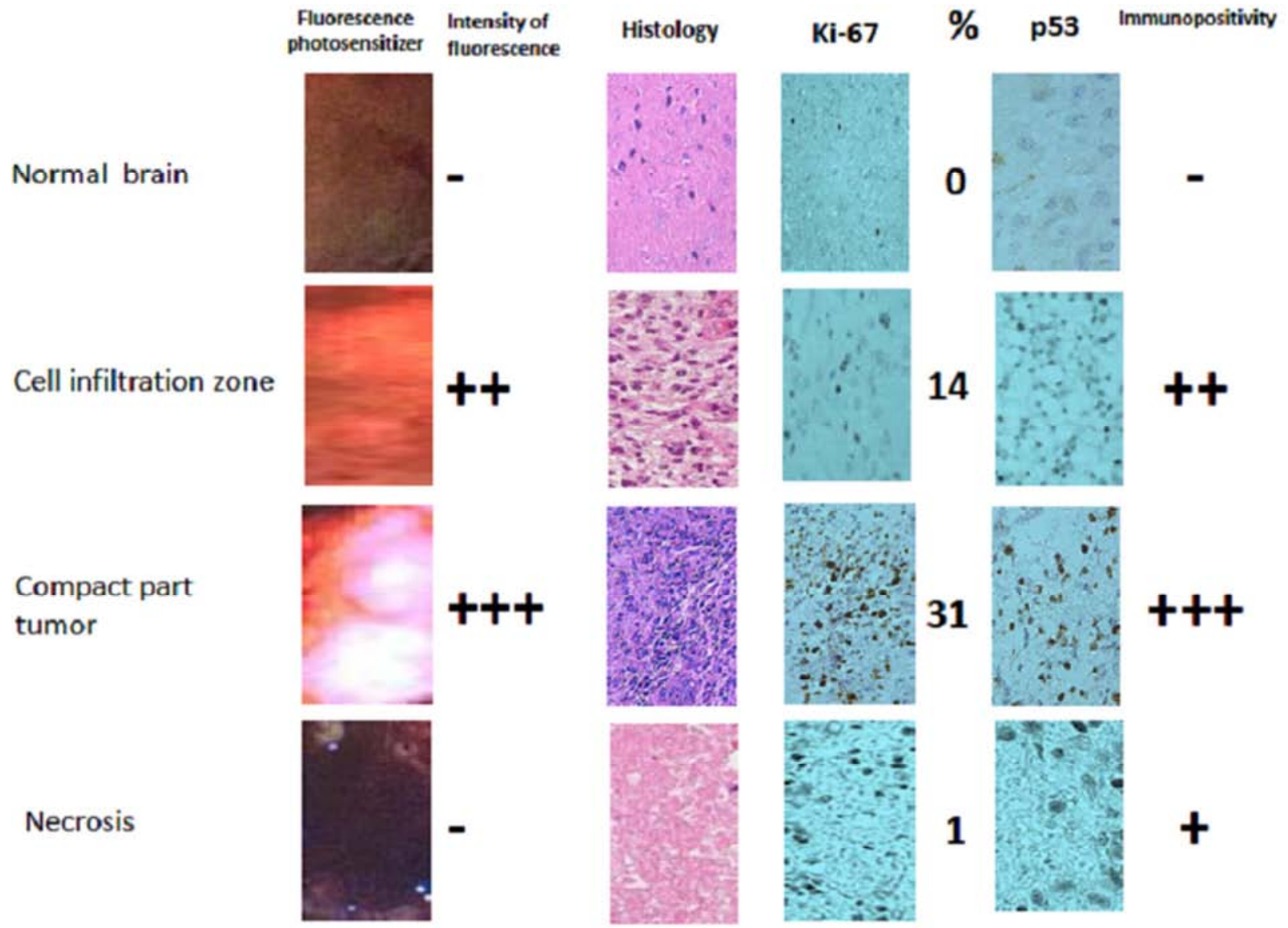

Figure 6. Comparative analysis of fotoditazine fluorescence with histological results (areas of visible fluorescence; intensity of fluorescence; histology with hematoxylin and eosin; Ki-67 (MIB-1, related to proliferation), P53 (TP53, related to apoptosis); immunopositivity).

Analysis of various glioma regions, including regions of differing fluorescence, showed that tumor necrosis zones corresponded with weak $(4.1 \%)$ or no $(94.7 \%)$ fluorescent signal. In conjunction with histological analysis, different regions of glioblastoma were analyzed, and it was found that tumor necrosis zones corresponded to an absence of fluorescence $(94.7 \%)$, or very weak fluorescence $(4.1 \%)$. Very bright fluorescence was detected in the areas of tumor infiltration $(91.5 \%)$.

In tumors, visible fluorescence was seen $83.3 \%$ of solid regions. In glioma biopsies from tumors (Grades II-IV), IHC (immunohistochemical) analysis of fluorescence-negative and fluorescence-positive samples showed correlations between the histologically-determined level of malignancy and fluorescence. In particular, the markers Ki-67 (MIB-1, related to proliferation) and P53 (TP53, related to apoptosis) both showed direct correlations to intensity and malignancy. In fluorescence-positive gliomas, a higher proliferation rate and a lower degree of differentiation were characteristic of higher malignancy, as shown in Table 3. 
Table 3. Distribution of visible fluorescence in glial tumor biopsies (Grades II-IV).

\begin{tabular}{llll}
\hline \multirow{2}{*}{ Criterion } & Characteristic & \multicolumn{2}{l}{ Fotoditazine-mediated indication } \\
\cline { 3 - 4 } & & Fluorescence-negative & Fluorescence-positive \\
\hline Patients & no & $2(100 \%)$ & $27(100 \%)$ \\
MRI* & patchy/faint & $1(25 \%)$ & $4(14.8 \%)$ \\
& focal & $1(25 \%)$ & $7(25.9 \%)$ \\
Grade & II & $1(25 \%)$ & $16(59.3 \%)$ \\
& III & $1(25 \%)$ & $4(14.8 \%)$ \\
Histopathological criteria & IV & $2(50 \%)$ & $10(37 \%)$ \\
& & & $13(48.2 \%)$ \\
Mitotic cells & none & $3(75 \%)$ & $2(7.5 \%)$ \\
& few & $1(25 \%)$ & $12(44.4 \%)$ \\
Nuclear pleomorphism & many & $0(0 \%)$ & $13(48.1 \%)$ \\
& low & $2(50 \%)$ & $2(7.4 \%)$ \\
Microvascular proliferation & moderate & $1(25 \%)$ & $17(63 \%)$ \\
& high & $1(25 \%)$ & $8(29.6 \%)$ \\
Necrosis & no & $4(100 \%)$ & $22(81.5 \%)$ \\
Ki-67 (MIB-1)** & yes & $0(0 \%)$ & $5(18.5 \%)$ \\
p53*** & yes & $3(75 \%)$ & $24(88.9 \%)$ \\
\hline
\end{tabular}

* contrast-enhanced magnetic resonance imaging; **Ki-67 (MIB-1, related to proliferation), ***P53 (TP53, related to apoptosis).

In 5 patients ( 2 in Grade III and 3 in Grade IV), perifocal tumor zones were studied. Specifically, tissue sites corresponding to different zones of fluorescent intensity were analyzed. Intense fluorescence in the perifocal zone corresponded to: a higher degree of anaplasia; higher TP53 and Ki-67 indices; and to higher levels of vascular endothelial growth factor (figure 7).
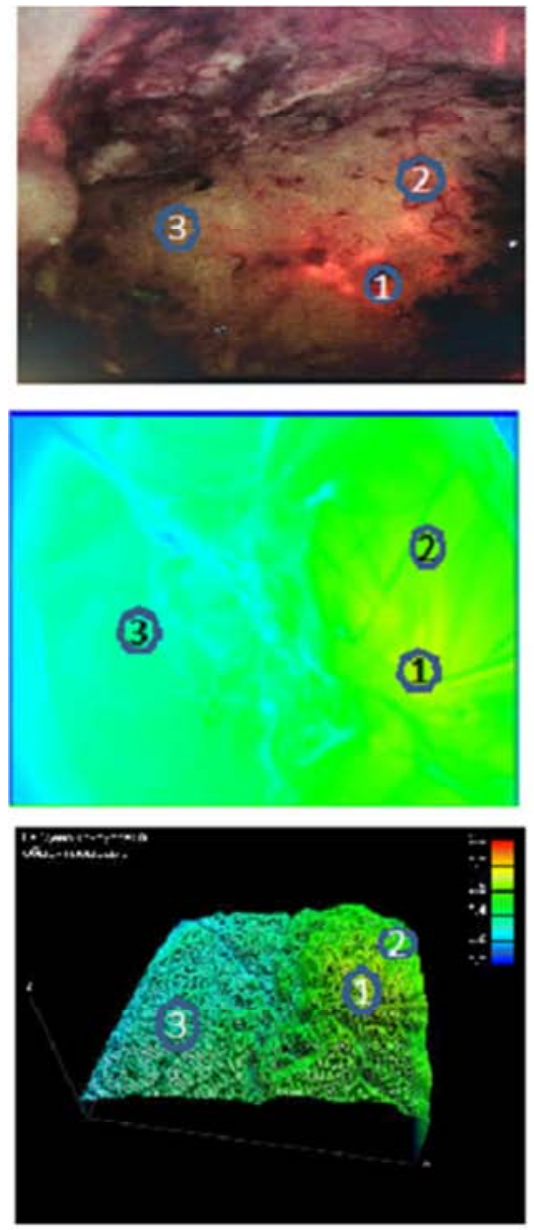

Hernatoxylin eosin

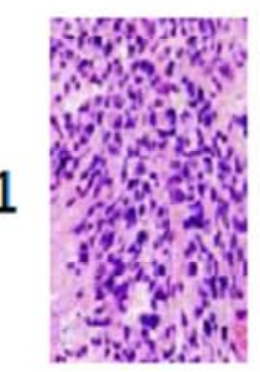

2

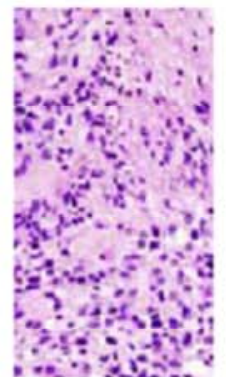

2

3
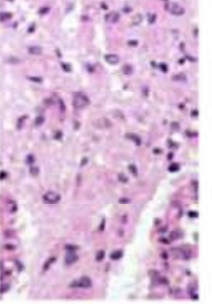

K0-67
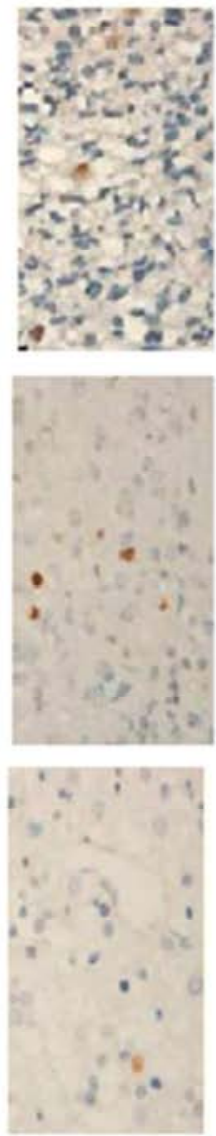

TP 53
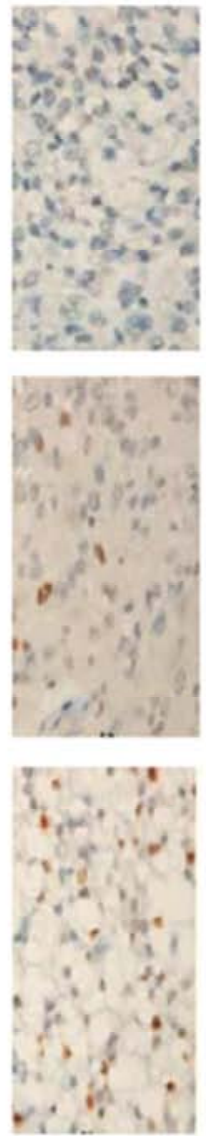

VEGF
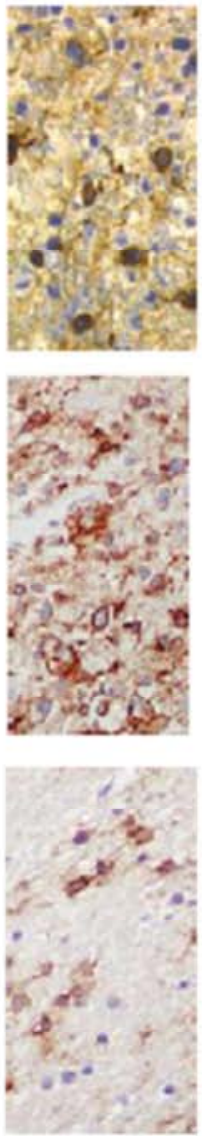

Figure 7. Comparative analysis of glioblastoma perifocal zone fluorescence in relation to immunohistochemical data. $1=$ area of intense fluorescence; $2=$ region of weak fluorescence; 3 = region with absence of fluorescence. (hematoxylin and eosin, Ki-67, TP53, VEGF-vascular endothelial growth factor). 
Comparative analysis, investigating the sensitivity and specificity of fluorescence-guided resection, showed that, in Grade I-II gliomas, the sensitivity of the surgical method was $68.3 \%$, and its specificity was $60.1 \%$. In Grade III-IV gliomas, the sensitivity of the method was $85.4 \%$, and its specificity was $76.2 \%$. The evaluation of the method's sensitivity and specificity, in comparison with the glial tumor biopsy specimens, is presented in Table. 4.

Table 4. Sensitivity and specificity of using visible fluorescence, in comparison with multiple glial tumor biopsies.

\begin{tabular}{lll}
\hline $\begin{array}{l}\text { Visible } \\
\text { fluorescence }\end{array}$ & $\begin{array}{l}\text { Sensitivity (biopsy } \\
\text { with a tumor) }\end{array}$ & $\begin{array}{l}\text { Specificity (biopsy tissue } \\
\text { with intact tissue) }\end{array}$ \\
\hline Grade II & $8 / 11(72.7 \%)$ & $2 / 5(60.0 \%)$ \\
Grade III & $15 / 18(83.3 \%)$ & $2 / 6(66.7 \%)$ \\
Grade IV & $21 / 24(87.5 \%)$ & $1 / 7(85.7 \%)$ \\
\hline
\end{tabular}

Among low-grade gliomas, oligodendrogliomas, diffuse astrocytomas, and gliomas with the presence of a cystic component demonstrated the highest fotoditazine accumulation capacities. Gross total resection (GTR) was defined as the removal of at least $90 \%$ of the contrasting part of the tumor, as determined by MRI performed within 24 hours of the completion of surgery. The degree to which GTR was achieved using intraoperative fluorescent navigation is presented in Table 5 .

Table 5. Achievement of GTR using fotoditazine intraoperative fluorescent navigation.

\begin{tabular}{ll}
\hline Grade & Level of resection (GTR) \\
\hline I $(\mathrm{n}=2)$ & $77.5 \%$ \\
II $(\mathrm{n}=5)$ & $81.1 \%$ \\
III $(\mathrm{n}=9)$ & $93.8 \%$ \\
IV $(\mathrm{n}=15)$ & $97.5 \%$ \\
\hline
\end{tabular}

Thus, on average, the degree to which GTR was achieved was $79.3 \%$ in Grade I-II glioma surgical interventions. In Grade III-IV gliomas - $95.6 \%$ was achieved.

\section{Discussion}

This study proves the effectiveness of using fluorescentlycontrolled resection in brain surgery for glioma. The method demonstrated here permits more thorough resection of the tumor mass; such surgical refinement will likely lead to improved patient prognosis and outcomes, including extension of the disease-free period. The use of fotoditazine to create and visualize fluorescence allows the surgeon to more accurately distinguish the edges of glioma intraoperatively. A meta-analysis of the literature on this topic also indicates the high effectiveness of this technique in comparison with traditional glioma microsurgery $[1,2,6]$. FGR is an inexpensive technique. The disadvantage of the method is availability of microscopes with the necessary optics for implementation, skin photosensitivity, hypotension and the limitation of its use in color blind surgeons.

\section{Conclusions}

Increasing the degree of resection of glial brain tumors can prolong survival and delay the recurrence. Neurosurgeons have to deal with the problem of maximizing the removal of brain cancer while maintaining the surrounding normal brain (especially eloquent areas, for example, motor, speech and sensory areas), as well as the difficulty of distinguishing a diffusely growing glial tumor from surrounding normal cells. The fluorescent navigation during brain surgery for supratentorially localized glial tumors allows the surgeon to not only clarify the size and location of the corticotomy. It also permits better understanding of the tumors' infiltrative (spread, invasive) status, thereby enabling more appropriate decision making regarding the boundaries of resection. Intraoperative fluorescent imaging with fotoditazine is a highly effective, sensitive, and specific method which permits glial tumors of various histologic types to be resected more completely (GTR) and effectively. The used fotoditazine drug, produced domestically in Russia, for fluorescent diagnosis in cases of brain tumor of glioma is safe for patients provided that appropriate selection criteria are used and guarantees for subsequent exposure to light are observed during the 24-hour postoperative period. Although most patients with glioma managed to achieve a significant GTR, in most cases, patients underwent photodynamic therapy to influence the remaining tumor cells. Some patients took further adjuvant therapy.

\section{Conflict of Interest}

The authors declare no conflict of interest.

\section{References}

[1] Eljamel S. 5-ALA Fluorescence Image Guided Resection of Glioblastoma Multiforme: A Meta-Analysis of the Literature. Int J Mol Sci. 2015; 16 (5): 10443-56.

[2] Su X, Huang QF, Chen HL, Chen J. Fluorescence-guided resection of high-grade gliomas: A systematic review and meta-analysis. Photodiagn Photodyn Ther. 2014; 1: 451-8.

[3] Della Puppa A, De Pellegrin S, d'Avella E, Gioffrè G, Rossetto M, Gerardi A, et al. 5-aminolevulinic acid (5-ALA) fluorescence guided surgery of high-grade gliomas in eloquent areas assisted by functional mapping. Our experience and review of the literature. Acta Neurochir. 2013; 155: 965-72.

[4] Jacquesson T, Ducray F, Maucort-Boulch D, Armoiry X, Louis-Tisserand G, Mbaye M. Surgery of high-grade gliomas guided by fluorescence: A retrospective study of 22 patients. Neurochirurgie 2013; 59: 9-16.

[5] Liu JT, Meza D, Sanai N. Trends in fluorescence imageguided surgery for gliomas. Neurosurgery 2014; 75 (1): 61-71.

[6] Rynda AY, Olyushin VE, Rostovtsev DM. FluorescenceGuided Resection of Glioma - literature review. Russian Neurosurgical Journal named after professor A. L. Polenov 2018; X (1): 91-101. 
[7] Acerbi F, Broggi M, Eoli M, Anghileri E, Cuppini L, Pollo B, et al. Fluorescein-guided surgery for grade IV gliomas with a dedicated filter on the surgical microscope: Preliminary results in 12 cases. Acta Neurochir. 2013; 155: 1277-86.

[8] Lau D, Hervey-Jumper SL, Chang S, Molinaro AM, McDermott MW, Phillips JJ, et al. A prospective Phase II clinical trial of 5-aminolevulinic acid to assess the correlation of intraoperative fluorescence intensity and degree of histologic cellularity during resection of high-grade gliomas. $\mathbf{J}$ Neurosurg. 2016; 124 (5): 1300-9.

[9] Jaber M, Wölfer J, Ewelt C, Holling M, Hasselblatt M, Niederstadt $\mathrm{T}$, et al. The value of 5-aminolevulinic acid in low-grade gliomas and high-grade gliomas lacking glioblastoma imaging features: an analysis based on fluorescence, magnetic resonance imaging, 18F-fluoroethyl tyrosine positron emission tomography, and tumor molecular factors. Neurosurgery 2016; 78 (3): P. 401-11.

[10] Bo Chen, Haifeng Wang, Pengfei Ge, Jingwei Zhao, Wenchen $\mathrm{Li}$, Huizi $\mathrm{Gu}$, et al. Gross total resection of glioma with the intraoperative fluorescence-guidance of fluorescein sodium. Int J Med Sci. 2012; 9: 708-14.

[11] Chung IW, Eljamel S. Risk factors for developing oral 5aminolevulinic acid-induced side effects in patients undergoing fluorescence guided resection. Photodiagn Photodyn Ther. 2013; 10: 362-7.

[12] Sadahiro Kaneko, Sadao Kaneko. Fluorescence-Guided Resection of Malignant Glioma with 5-ALA. International Journal of Biomedical Imaging 2016; 11 (2): 1-11.

[13] Valdés PA, Kim A, Brantsch M, Niu C, Moses ZB, Tosteson $\mathrm{TD}$, et al. Delta-aminolevulinic acid-induced protoporphyrin IX concentration correlates with histopathologic markers of malignancy in human gliomas: the need for quantitative fluorescence-guided resection to identify regions of increasing malignancy. Neuro Oncol. 2011; 13: 846-56.

[14] Zheng H, Songsheng Shi, Haixia Qiu, Desheng Li, Jian Zou, Shaoshan $\mathrm{Hu}$. Fluorescence-guided resection of brain tumor: review of the significance of intraoperative quantification of protoporphyrin IX fluorescence. Neurophotonics 2017; 4 (1): P. 011011.

[15] Neira JA, Ung TH, Sims JS, Malone HR, Chow DS, Samanamud JL, et al. Aggressive resection at the infiltrative margins of glioblastoma facilitated by intraoperative fluorescein guidance. Journal of Neurosurgery 2017; 127 (1): 111-22.

[16] Hauser SB, Kockro RA, Actor B, Sarnthein J, Bernays RL. Combining 5-aminolevulinic acid fluorescence and intraoperative magnetic resonance imaging in glioblastoma surgery: a histology based evaluation. Neurosurgery 2016; 78: 475-83.

[17] Eljamel MS, Mahboob SO. The effectiveness and costeffectiveness of intraoperative imaging in high-grade glioma resection; a comparative review of intraoperative ALA, fluorescein, ultrasound and MRI. Photodiagnosis Photodyn Ther. 2016; 16: 35-43.

[18] Louis DN. World Health Organization Histological Classification Classification of Tumours of the Central Nervous System. Lyon, France: International Agency for Research on Cancer. 2016.

[19] Rynda AY, Olyushin VE, Rostovtsev DM. Photodynamic therapy of cerebral glioma - long term survival. Vestnik Rossiiskoi Voenno-Medicinskoi Academii 2017; 2 (58): 6872. 